\title{
THE METHODS TO SHAPE THE UPPER EYELID FOLD
}

\author{
Hoang Thi Phuong Lan ${ }^{1,}$, Nguyen Bac Hung ${ }^{2}$ \\ ${ }^{1}$ Duc Giang General Hospital, \\ 2108 Military Central Hospital
}

With nearly half of the Asian population having no obvious double eyelid, cosmetic surgery to create double eyelids or create upper eyelid fold is the most common cosmetic procedure requested in Asia, including Vietnam. In this article, we summarized and explored the techniques for creating upper eyelid fold for those who have no crease or not apparent crease. We also review the development of upper eyelid surgery throughout history in Asia and analyze the technical and risks of each eyelid surgical technique.

Keywords: Double eyelid, upper eyelid fold.

\section{INTRODUCTION}

The eyelids are important visual parts of the anatomy. Beside the acknowledged function of the morphology of the eyelids, the upper eyelid fold plays an important part in creating a harmonious look in the perception of East Asians. It is generally believed that the big round double-eyelids are indicators of beauty and youthfulness. The eyelid morphologies such as single eyelids and low eyelid crease (low-seated, nasally tapered, including hidden fold), combined with so-called Epicanthus palpebronasal fold are often considered to be less aesthetic and contribute to less expressive facial emotions. Therefore, the apparent, symmetrical upper eyelid fold, i.e., double eyelids, are considered more aesthetically pleasing than the single eyelids. However, half of the Asian population, including Vietnamese, is born without double-eyelids. Brian demonstrated that only about $50 \%$ of Asians have eyelid folds. Those eyelid folds can be the complete upper eyelid, low eyelid

Corresponding author: Hoang Thị Phuong Lan,

Duc Giang General Hospital

Email: hoanglanthammyducgiang@gmail.com

Received: 12/04/2021

Accepted: 20/05/2021 crease, intermittent eyelid, or multiple eyelid folds. Additionally, the more popular features of the Asian eyelid are either single or Epicanthus palpebronasal fold. A significant number of Asians with single eyelids, incomplete eyelids, asymmetrical and small upper eyelids are thus often advised to undergo double-eyelids surgery.

\section{CONTENT}

\section{The development of upper eyelid surgical techniques}

According to some Japanese medical literature, in 1896, Kotaro Mikamo became the first Japanese surgeon to introduce the surgical technique of knot-suturing the upper eyelid fold to artificially create double eyelids. Mikamo performed the operation on a Japanese woman who had one eye with a double eyelid and the other was a monolid. He performed the technique of suturing through the full thickness of the single eyelid with three 4.0 braided silk sutures.

Thirty years later, in 1926, the suture ligation surgical technique for the upper eyelid fold was introduced by Uchida. Uchida performed over 1523 cases of repairing upper eyelid folds in 
396 males and 444 females. The eyelid folds were shaped like a slightly rounded fan. ${ }^{2}$ Upper eyelid fold surgery was first mentioned in 1929 when Maruo reported techniques for both incision and suturing to create upper eyelid folds. Maruo designed the crease across the upper eyelid fold with the height between the eyelid margin and the crease which was $7 \mathrm{~mm} .^{8}$

In 1933, Hata reported the suture ligation methods producing greater eyelid crease's height of $10 \mathrm{~mm}$. He used three braided silk sutures to pass through the eyelid cartilage to the skin and fix the sutures on the skin surface with small buttons. ${ }^{3}$

In a 1938 thematic report, Hayashi described two methods of eyelid crease shaping. With knot-suture methods by which 3 stitches were placed in each of the upper eyelids. ${ }^{4}$

In 1947, Inoue presented a technique to remove the upper eyelid's subcutaneous connective tissue layers between the incision and the eyelid edge. 5.0 braided silk suture was used to close the incision passing through skin to the cartilage and back to the skin. ${ }^{9}$

In 1950, Mitsui introduced another upper eyelid forming method. He continued to develop techniques to artificially create eyelid crease ${ }^{10}$ by taking risks in removing the pre-cartilage connective tissue including the orbicular muscle of the eye, the orbital fat and the pre-cartilage fat.

In 1954, Filipino ophthalmic surgeon Sayoc described a double eyelid blepharoplasty, making an incision 1-3 $\mathrm{mm}$ to the pre-cartilage of orbicular muscle of eye. ${ }^{11}$ Eyelid fold incision was closed in the skin-cartilage suturing style by burying either 6.0 silk sutures or catgut sutures. Those sutures could be removed after 5 days.

In 1960, Fernandez, American Plastic Surgeon presented a double eyelid forming method that removed an $8-10 \mathrm{~mm}$ ellipse of skin, 3-5mm ellipse of orbicular muscle, the orbital septum, and the orbital fat. ${ }^{13}$ This was considered to be a more radical method compared to the previous methods.

In 1963, Boo-Chai described a technique which was similar to that of Mitsui's. ${ }^{14}$

In 1985, in China, Song R.Y, Song Y.G described three types of double eyelid fold surgery: the hanging method, the skin incision method, and the skin grafting method. ${ }^{15}$

In 1992, in Korea, a surgical technique to create an upper eyelid fold that was not fixed to the cartilage was introduced by Bang Y.H. He argued that the importance of the amount of soft tissues may outweigh that of the enlargement of the eyelid levator muscles when it comes to eyelid crease formation. Accordingly, he performed double eyelid surgeries by removing excess soft tissue without fixing the skin to the eyelid levator muscle membrane or cartilage. ${ }^{16}$

In 1995, author Shiao I.S came up with a double eyelid surgical technique for people of Eastern descent. It was a simple non-incisional method using U-shaped sutures. ${ }^{5}$ The benefits of this technique included: No incision and a cosmetically desired crease is expected in the upper eyelid cartilage with the enhancement of sutures.

In 1995, Shiao I.S presented a method using the twin-needle suture, tying the eyelid fold by a U-shaped fashion. The advantages of this technique included no need to turn the eyelid outwards and creating an aesthetically desired crease of the upper eyelid cartilage thanks to using suture of greater strength. ${ }^{5}$

In 1997, Megumi Y. introduced the U-suture method with the sling technique, removing fat from the upper eyelid through the conjunctiva without damaging the upper eyelid skin ${ }^{6}$.

In 1999, Jung Park reported on the technique of attaching the eyelid sphincter (not skin) to the 
upper eyelid levator muscle ${ }^{18}$.

In 2013, Kyung-Chul Moon reported the procedure of upper eyelid crease formation by using single-knot and buried non-incisional technique at one position?

\section{The methods to shape the upper eyelid} fold

The principal methods for creating the double eyelid can be broadly divided into the suture method and the incision method.

\section{The suture method}

The advantage of this technique is being simpler, faster, less invasive, easier to care for, and postoperatively more relaxing. However, the downside is that it is limited to people with excess skin of the upper eyelid. In addition, the eyelid crease can often become less visible or disappear over time. However, nowadays, some other techniques for artificially creating double eyelid folds are widely used. The suturing methods to shape the upper double eyelid are divided into two groups: the external suturing on the skin and buried sutures under the skin.

\section{The method of external sutures on the skin}

The method of external sutures consisted of three sub-groups: ${ }^{17}$

- Suture fixation of the skin to cartilage: This method is the oldest method. This is a simple method that creates a good, fixed position. However, this method has the potential risk of cutting nutrition to the cartilage and causing eyelid cartilage deformation, therefore this technique was no longer used.

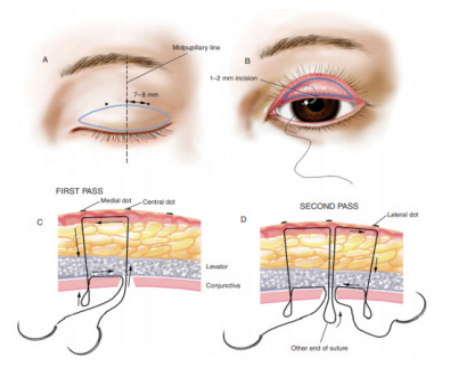

Figure 1. The suture methods ${ }^{18}$
- Suture fixation of the skin to levator: In this technique, the eyelid levator muscle and Muller's muscles are attached to the upper edge of the upper ciliary cartilage. This technique of suturing on a muscle is the oldest. However, this method is also no longer used due to scars created in the upper eyelid skin.

- Suture fixation to the conjunctiva: This method requires an experienced surgeon. The advantage of the technique is that it causes less postoperative swelling. Currently, this technique is rarely used because it is difficult to carry out, difficult to remove sutures, and the risk of corneal irritation and conjunctivitis is great.

The method of buried sutures under the skin

This method is actually a combined method using eyelid incision and buried sutures. It is possible to combine the ocular fat removal through the eyelid incision. These methods are divided into two small groups: spaced buried suturing and continuous buried suturing.

\section{The incision method}

The incision method can be conducted via a standard-length blepharoplasty incision or a short incision. The traditional Asian blepharoplasty incision is made along the length of the desired fold. A short-incision technique with a $10 \mathrm{~mm}$ to $13 \mathrm{~mm}$ incision can not only reduce scarring and recovery time but also provide a natural skin crease. This technique is less suitable for those who require skin excision or significant pretarsal orbicularis excision.

\section{The standard-length incision method}

In addition to the advantage of active and appropriate double eyelid formation, the full upper eyelid forming method is applicable for all upper eyelid characteristics. The combination of removing excess eyelid skin and orbital fat, suturing the skin to the levator muscles, creating a palpebronasal fold always maximizes 
the shape and size of the upper eyelid fold. Specifically, the eyelids are considered to be cosmetically longer and wider (larger round eyes), and the eyelid folds being larger, deeper, clearer, more evenly and long-lasting. The external incision method is more controlled and permanent. This method can remove a variable amount of skin depending on the patient's needs. It also resects some orbicularis oculi usually several millimeters along the preseptal and the pretarsal segments. The orbital septum is opened superiorly and, depending on the situation, a variable amount of preaponeurotic fat may be trimmed, but never completely
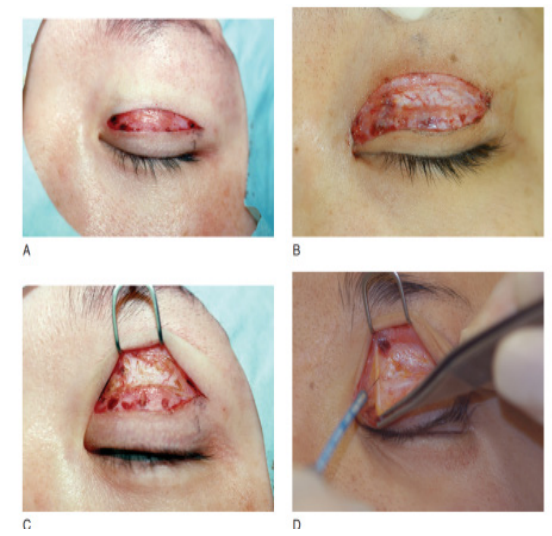

\section{Figure 2. The standard-length incision methods $^{18}$}

\section{CONCLUSION}

The exact determination of the double eyelid forming surgical method for each Vietnamese individual has not been systematically studied. There are a variety of methods, each with its own advantages; the choice of double-eyelid-foldsshaping method depends on the subjectively aesthetic perception and experience of the surgeon as well as patient's request.

\section{REFERENCES}

1. Shiao, I-Sen. "Oriental double eyelid: a simplified nonincisional technique using the removed. The lid crease-enhancing sutures are placed skin-levator aponeurosis-skin. To give a dynamic, superficial crease was created and no suture materials was buried.

\section{The short incision methods:}

Partial-Incision double eyelidplasty can remove excess upper eyelid skin and orbital fat. ${ }^{19,20}$ The main advantage of this surgical technique is to minimize invasive surgery and visible scarring to the upper eyelid area. Rohrich ${ }^{19}$ proposed five-step procedure which are key in the correction of upper lid age-related changes and provide a reliable and reproducible method of achieving excellent results.
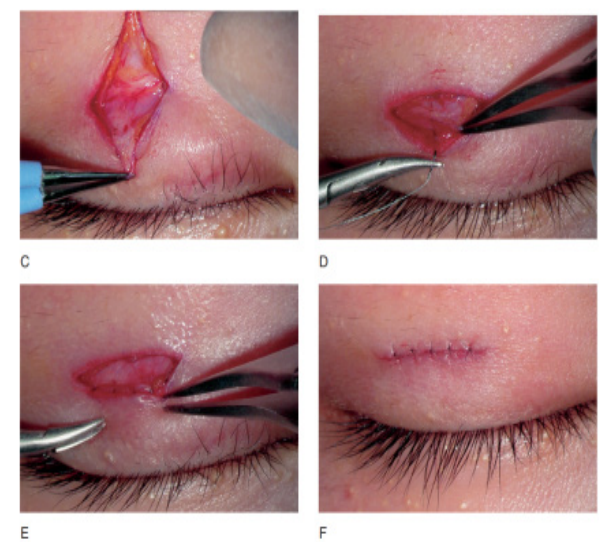

Figure 3. The short incision methods ${ }^{18}$

twin-needle suturer." Plastic and reconstructive surgery 96.1 (1995): 179-182.

2. Homma, Ken-ichi, Yasuo Mutou, Hideo Mutou, Kyori Ezoe, and Tatsuya Fujita. "Intradermal stitch blepharoplasty for Orientals: does it disappear?" Aesthetic plastic surgery 24, no. 4 (2000): 289-291.

3. Moon, Kyung-Chul, Eul-Sik Yoon, and Jun-Mun Lee. "Modified double-eyelid blepharoplasty using the single-knot continuous buried non-incisional technique." Archives of plastic surgery 40, no. 4 (2013): 409. 
4. Maruo, M. "Plastic reconstruction of a double eyelid." Jpn Rev Clin Ophthalmol 24 (1929): 393.

5. Inoue, S. "The double eyelid operation." Jpn Rev Clin Ophthalmol 27 (1947): 306.

6. Mitsui, Y. "Plastic construction of a" double-eyelid." Jpn Rev Clin Ophthalmol 44 (1950): 19.

7. Sayoc BT: Plastic construction of the superior palpebral fold. Am J Ophthalmol, 38:556-559, 1954.

8. Millard, D. R., Jr.: Oriental peregrinations. Plast \& Reconst Surg, 16:319, 1955.

9. Fernandez LR: Double eyelid operation in the Oriental in Hawaii. Plast Reconstr Surg, 1960;25:257-264

10. Boo-Chai K: Plastic construction of the superior palpebral fold. Plast Reconstr Surg, 1963; 31:74-78.

11. Song, Ru-yao, and Ye-guang Song. "Double eyelid operations." Aesthetic plastic surgery 9, no. 3 (1985): 173-180.

12. Bang, Yoo Hyun. "The double-eyelid operation without supratarsal fixation." Plastic and reconstructive surgery 88, no. 1 (1992): 12-7.

13. Megumi, Yoshikazu. "Double eyelid procedure by removal of transconjunctival orbital fat and buried sutures combined with sling technique to avoid wounding the eyelid." Aesthetic plastic surgery 21, no. 4 (1997): 254-257.

14. Park, Jung I. "Orbicularis-levator fixation in double-eyelid operation." Archives of facial plastic surgery 1, no. 2 (1999): 90-95.

15. Chen WP. Concept of triangular, trapezoidal and rectangular debulking of eyelid tissues: application in Asian blepharoplasty. Plast Reconstr Surg, 1996;97: 212-8.

16. Scawn, Richard, Naresh Joshi, and Yoon-Duck Kim. "Upper lid blepharoplasty in Asian eyes." Facial Plastic Surgery 26, no. 02 (2010): 086-092.

17. Chen W, (2010), "Upper Eyelid of Asians Without Crease". Color Atlas of Cosmetic Oculofacial Surgery"; 13-134.

18. Jung Park (2007), "Asian blepharoplasty". Asian facial cosmetic surgery; 1-116.

19. Rohrich, Rod J. M.D.; Villanueva, Nathaniel L. M.D.; Afrooz, Paul N. M.D. 2018

20. "Refinements in Upper Blepharoplasty: The Five-Step Technique". Plastic and Reconstructive Surgery: Volume 141 - Issue 5 p 1144-1146. 